\title{
A FUNDAMENTAL MODULAR IDENTITY AND SOME APPLICATIONS
}

\author{
RICHARD BLECKSMITH, JOHN BRILLHART, AND IRVING GERST \\ Dedicated to the memory of D. H. Lehmer
}

\begin{abstract}
We prove a six-parameter identity whose terms have the form $x^{\alpha} T\left(k_{1}, l_{1}\right) T\left(k_{2}, l_{2}\right)$, where $T(k, l)=\sum_{-\infty}^{\infty} x^{k n^{2}+l n}$. This identity is then used to give a new proof of the familiar Ramanujan identity $H(x) G\left(x^{11}\right)-$ $x^{2} G(x) H\left(x^{11}\right)=1$, where $G(x)=\prod_{n=0}^{\infty}\left[\left(1-x^{5 n+1}\right)\left(1-x^{5 n+4}\right)\right]^{-1}$ and $H(x)=\prod_{n=0}^{\infty}\left[\left(1-x^{5 n+2}\right)\left(1-x^{5 n+3}\right)\right]^{-1}$. Two other identities, called "balanced $Q^{2}$ identities", are also established through its use.
\end{abstract}

\section{INTRODUCTION}

It is our purpose in this paper to prove a fundamental identity, whose terms are a certain type of modular form, and then to demonstrate its use in proving other identities. This general identity, its proof, and the three applications of the identity that are made here were discovered by a mixture of theoretical studies and computer analyses of particular identities. This work is different from that presented in [2-4], where the identity to be proved was first discovered by a computer search and then was proved by a computer-assisted, theoretical argument.

We will use the two single-variable $T$-functions $T_{0}$ and $T_{1}$ (cf. [4, equation (2)]), defined by

$$
T(k, l)=T_{0}(k, l) \stackrel{\text { def }}{=} \sum_{-\infty}^{\infty} x^{k n^{2}+l n}=\prod_{n=1}^{\infty}\left(1-x^{2 k n}\right)\left(1+x^{2 k n-k+l}\right)\left(1+x^{2 k n-k-l}\right)
$$

and

$$
T_{1}(k, l) \stackrel{\text { def }}{=} \sum_{-\infty}^{\infty}(-1)^{n} x^{k n^{2}+l n}=\prod_{n=1}^{\infty}\left(1-x^{2 k n}\right)\left(1-x^{2 k n-k+l}\right)\left(1-x^{2 k n-k-l}\right) .
$$

We call an identity a " $T^{2}$ identity" if each of its terms has the form

$$
x^{\alpha} T_{\epsilon_{1}}\left(k_{1}, l_{1}\right) T_{\epsilon_{2}}\left(k_{2}, l_{2}\right),
$$

where $\epsilon_{1}$ and $\epsilon_{2}$ are 0 or 1 . We also say that a $T^{2}$ identity is "balanced" if the first component pair $\left(k_{1}, k_{2}\right)$ in each of its terms is the same.

Received by the editor July 27, 1992.

1991 Mathematics Subject Classification. Primary 05A19.

Key words and phrases. Triple and quintuple product, modular identity, balanced $T^{2}$ and $Q^{2}$ identity. 
In $\S 2$ we will prove the fundamental identity (Theorem 1). It will then be used in $\S 3$ to give a new proof of a familiar identity of Ramanujan (Theorem 2) and in $\S 4$ to provide proofs for two interesting identities, called "balanced $Q^{2}$ identities" (Theorems 3 and 4). In all of these proofs the fundamental identity is specialized to produce a small family of identities by assigning sets of values to its parameters. The identity in question is then established by showing it to be a linear combination of the identities in this family.

These three proofs illustrate a tentative proof method of three steps: (1) If possible, transform the given identity into a $T^{2}$ identity; (2) Balance this identity using the expansion formula in [4, p. 779]; (3) Determine if and how the fundamental formula can be used to generate a small family of identities in terms of which the given identity can be expressed as a linear combination.

\section{THE FUNDAMENTAL IDENTITY}

The following general identity, which is of interest in its own right, is also important in generating special sets of identities from which proofs of other identities can be made.

In working with $T$-functions, it is often important to re-index their sums. This can be accomplished by simple transformation rules, which are also useful in putting $T$ in "reduced" form. (The function $T_{\epsilon}(k, l)$ is in "reduced form" when $0 \leq l \leq k$ (cf. [4, p. 780]).) Then, for $\epsilon \in\{0,1\}$, there is

the negative rule $[4,(13)]$ :

$$
T_{\epsilon}(k,-l)=T_{\epsilon}(k, l),
$$

the single-step formula $[4,(14)]$ :

$$
x^{\alpha} T_{\epsilon}(k, l)=(-1)^{\epsilon} x^{\alpha-(l-k)} T_{\epsilon}(k, 2 k-l),
$$

and

the general transformation formula: If $l=2 k q+r$, where $q \in Z$, then

$$
x^{\alpha} T_{\epsilon}(k, l)=(-1)^{\epsilon q} x^{\alpha-q^{2} k-q r} T_{\epsilon}(k, r) .
$$

Proof of (2.3). We have that

$$
\begin{aligned}
x^{\alpha} T_{\epsilon}(k, l) & =x^{\alpha} \sum_{-\infty}^{\infty}(-1)^{\epsilon n} x^{k n^{2}+(2 k q+r) n} \\
& =x^{\alpha} \sum_{-\infty}^{\infty}(-1)^{\epsilon(n-q)} x^{k(n-q)^{2}+(2 k q+r)(n-q)} \\
& =(-1)^{\epsilon q} x^{\left(\alpha-q^{2} k-q r\right.} T_{\epsilon}(k, r) .
\end{aligned}
$$

Note, when we use $(2.3)$ to put $T(k, l)$ numerically into reduced form, we take $r$ to be in the interval $-k<r \leq k$, and then use (2.1) if necessary. Throughout the rest of this paper we will give all $T$-functions with numerical arguments in reduced form.

Theorem 1. Suppose that $m, u, v \in Z^{+}, e, f \in Q$, and $k \in Q^{+}$, where $u v<2 m$. Then 


$$
\sum_{n \in R_{m}} x^{\alpha_{n}} T\left(k_{1}, l_{1 n}\right) T\left(k_{2}, l_{2 n}\right)=\sum_{n \in R_{m}^{\prime}} x^{\alpha_{n}} T\left(k_{1}, l_{1 n}^{\prime}\right) T\left(k_{2}, l_{2 n}^{\prime}\right),
$$

where

$$
\alpha_{n}=\frac{2 v k}{m} n^{2}+2 e n, \quad k_{1}=u k, \quad k_{2}=(2 m-u v) v k,
$$

$$
\begin{aligned}
& \left\{\begin{array}{l}
l_{1 n}=\frac{2 u v k}{m} n+u e+f, \\
l_{2 n}=(2 m-u v)\left(\frac{2 v k}{m} n+e\right)-v f,
\end{array}\right. \\
& \left\{\begin{array}{l}
l_{1 n}^{\prime}=\frac{2 u v k}{m} n+u e-f, \\
l_{2 n}^{\prime}=(2 m-u v)\left(\frac{2 v k}{m} n+e\right)+v f,
\end{array}\right.
\end{aligned}
$$

and $R_{m}$ and $R_{m}^{\prime}$ are any complete residue systems $(\bmod m)$.

Proof. The proof rests on the following generalization of the expansion formula in [4, p. 779]. (The notation in [4] is changed here to suit the present proof. Note that the proof presented in [4] remains valid when we allow the parameters $k>0$ and $l$ in $T(k, l)=\sum_{-\infty}^{\infty} x^{k n^{2}+l n}$ to be rational.)

Let $a, b \in Z, \mu \in Z^{+},\left(\kappa_{1}, \lambda_{1}\right),\left(\kappa_{2}, \lambda_{2}\right) \in Q^{+} \times Q$, and $\epsilon_{1}, \epsilon_{2} \in\{0,1\}$. If the separability condition

$$
\kappa_{1} b=\kappa_{2} a(\mu-a b)
$$

is satisfied, then

$$
\begin{aligned}
& T_{\epsilon_{1}}\left(\kappa_{1}, \lambda_{1}\right) T_{\epsilon_{2}}\left(\kappa_{2}, \lambda_{2}\right) \\
& \quad=\sum_{n \in R_{\mu}}(-1)^{\epsilon_{2} n} x^{\kappa_{2} n^{2}+\lambda_{2} n} T_{\delta_{1}}\left(k_{1}, l_{1}(n)\right) T_{\delta_{2}}\left(k_{2}, l_{2}(n)\right),
\end{aligned}
$$

where

$$
\begin{array}{ll}
k_{1}=\kappa_{1}+\kappa_{2} a^{2}, & k_{2}=\kappa_{2} \mu(\mu-a b), \\
l_{1}(n)=\lambda_{1}-\lambda_{2} a-2 \kappa_{2} a n, & l_{2}(n)=(\mu-a b)\left(2 \kappa_{2} n+\lambda_{2}\right)+\lambda_{1} b, \\
\delta_{1}=\left(\epsilon_{1}+\epsilon_{2} a\right)(\bmod 2), & \delta_{2}=\left(\epsilon_{1} b+\epsilon_{2}(\mu-a b)\right)(\bmod 2),
\end{array}
$$

and $R_{\mu}$ is a complete residue system $(\bmod \mu)$.

In (2.8) substitute

$$
\begin{array}{ll}
\kappa_{1}=\frac{(2 m-u v) u k}{2 m}, & \kappa_{2}=\frac{v k}{2 m}, \\
\lambda_{1}=-f, & \lambda_{2}=e, \\
\epsilon_{1}=u \bmod 2, & \epsilon_{2}=1,
\end{array}
$$

and use the parameters $a=u, b=v$, and $\mu=2 m$. Note that (2.7) is satisfied, since by (2.9)

$$
\kappa_{1} v=\kappa_{2} u(\mu-u v)
$$


We then have that

$$
\begin{aligned}
& k_{1}=\kappa_{1}+\kappa_{2} a^{2}=\frac{(2 m-u v) u k}{2 m}+\frac{v k u^{2}}{2 m}=u k, \\
& k_{2}=\kappa_{2} \mu(\mu-a b)=(2 m-u v) v k, \\
& \delta_{1}=\left(\epsilon_{1}+\epsilon_{2} u\right) \bmod 2=0, \\
& \delta_{2}=\left(\epsilon_{1} v+\epsilon_{2}(2 m-u v)\right) \bmod 2=v\left(\epsilon_{1}+\epsilon_{2} u\right) \bmod 2=0 .
\end{aligned}
$$

Since $T=T_{0}$, the expansion is then

$$
T_{\epsilon_{1}}\left(\kappa_{1}, \lambda_{1}\right) T_{\epsilon_{2}}\left(\kappa_{2}, \lambda_{2}\right)=\sum_{n \in R_{2 m}}(-1)^{\epsilon_{2} n} x^{\alpha(n)} T\left(k_{1}, l_{1}(n)\right) T\left(k_{2}, l_{2}(n)\right)
$$

where (using $-l_{1}(n)$ by $(2.1)$ )

$$
\begin{aligned}
& \alpha(n)=\kappa_{2} n^{2}+\lambda_{2} n=\frac{v k}{2 m} n^{2}+e n \\
& l_{1}(n)=2 \kappa_{2} a n+\lambda_{2} a-\lambda_{1}=\frac{u v k}{m} n+u e+f \\
& l_{2}(n)=(\mu-a b)\left(2 \kappa_{2} n+\lambda_{2}\right)+\lambda_{1} b=(2 m-u v)\left(\frac{v k}{m} n+e\right)-v f .
\end{aligned}
$$

Now separate the sum on the right side of (2.11) into the difference of two sums,

$$
T_{\epsilon_{1}}\left(\kappa_{1}, \lambda_{1}\right) T_{\epsilon_{2}}\left(\kappa_{2}, \lambda_{2}\right)=S_{+}-S_{-}
$$

where

$$
\begin{aligned}
& S_{+}=\sum_{n \in R_{m}} x^{\alpha(2 n)} T\left(k_{1}, l_{1}(2 n)\right) T\left(k_{2}, l_{2}(2 n)\right) \text { and } \\
& S_{-}=\sum_{n \in R_{m}} x^{\alpha(2 n+1)} T\left(k_{1}, l_{1}(2 n+1)\right) T\left(k_{2}, l_{2}(2 n+1)\right) .
\end{aligned}
$$

Next, consider the expansion of the product

$$
\begin{aligned}
T_{\epsilon_{1}}\left(\kappa_{1},\right. & \left.-\lambda_{1}\right) T_{\epsilon_{2}}\left(\kappa_{2}, \lambda_{2}\right)=S_{+}^{\prime}-S_{-}^{\prime} \\
= & \sum_{n \in R_{m}^{\prime}} x^{\alpha(2 n)} T\left(k_{1}, l_{1}^{\prime}(2 n)\right) T\left(k_{2}, l_{2}^{\prime}(2 n)\right) \\
& -\sum_{n \in R_{m}^{\prime}} x^{\alpha(2 n+1)} T\left(k_{1}, l_{1}^{\prime}(2 n+1)\right) T\left(k_{2}, l_{2}^{\prime}(2 n+1)\right)
\end{aligned}
$$

where $l_{1}^{\prime}$ and $l_{2}^{\prime}$ are obtained from $l_{1}$ and $l_{2}$ by replacing $f$ by $-f$ in $(2.12)$. Since $T_{\epsilon_{1}}\left(\kappa_{1}, \lambda_{1}\right)=T_{\epsilon_{1}}\left(\kappa_{1},-\lambda_{1}\right)$, we have that

$$
S_{+}-S_{-}=S_{+}^{\prime}-S_{-}^{\prime}
$$

Now repeat the preceding development but with $\epsilon_{1}=\epsilon_{2}=0$ in (2.8). Since all the terms of the resulting expansion are the same as before, except that they 
are now all positive, it follows that

$$
T_{0}\left(\kappa_{1}, \lambda_{1}\right) T_{0}\left(\kappa_{2}, \lambda_{2}\right)=S_{+}+S_{-}
$$

and

$$
T_{0}\left(\kappa_{1},-\lambda_{1}\right) T_{0}\left(\kappa_{2}, \lambda_{2}\right)=S_{+}^{\prime}+S_{-}^{\prime}
$$

so

$$
S_{+}+S_{-}=S_{+}^{\prime}+S_{-}^{\prime}
$$

It then follows from $(2.14)$ to $(2.15)$ that $S_{+}=S_{+}^{\prime}$ and $S_{-}=S_{-}^{\prime}$. On the one hand, from (2.13) we have

$$
S_{+}=\sum_{n \in R_{m}} x^{\alpha_{n}} T\left(k_{1}, l_{1 n}\right) T\left(k_{2}, l_{2 n}\right)
$$

where from (2.12) we obtain

$$
\begin{aligned}
& \alpha_{n}=\alpha(2 n)=\frac{2 v k}{m} n^{2}+2 e n, \\
& l_{1 n}=l_{1}(2 n)=\frac{2 u v k}{m} n+u e+f, \\
& l_{2 n}=l_{2}(2 n)=(2 m-u v)\left(\frac{2 v k}{m} n+e\right)-v f,
\end{aligned}
$$

which is the left-hand side of (2.4). Similarly $S_{+}^{\prime}$ is the right-hand side of (2.4), so (2.4) is proved.

Remarks. 1. In (2.4) we can permit $\alpha_{n}$ to be negative, since negative powers of $x$ can be removed from the equation by multiplying through by an appropriate power of $x$.

2. We do not obtain another identity from the equation $S_{-}=S_{-}^{\prime}$, since this identity arises from (2.4) by replacing " $e$ " by " $e+\frac{v k}{m}$ " in (2.5) and (2.6), and multiplying the resulting identity by " $x^{e+(v k / 2 m) "}$.

\section{A NEW PROOF OF A RAMANUJAN IDENTITY}

In this section and the next we will prove three identities using Theorem 1. In each of these proofs a small family of identities is derived from (2.4) by giving certain sets of values to its parameters. The identity in question is then verified by showing it is a particular linear combination of these identities.

The first identity we will consider is a familiar result of Ramanujan.

Theorem 2 (Ramanujan [7, 9, 10, 1, 6, 8]). If

$$
G(x)=\prod_{n=0}^{\infty} \frac{1}{\left(1-x^{5 n+1}\right)\left(1-x^{5 n+4}\right)} \quad \text { and } \quad H(x)=\prod_{n=0}^{\infty} \frac{1}{\left(1-x^{5 n+2}\right)\left(1-x^{5 n+3}\right)}
$$

then

$$
H(x) G\left(x^{11}\right)-x^{2} G(x) H\left(x^{11}\right)=1 .
$$

Proof. The proof is in three steps.

(i) We begin by transforming (3.1) into a $T^{2}$ identity. For simplicity, write the infinite products in (3.1) in the abbreviated notation 


$$
\left(r_{1}, \ldots, r_{t}\right)_{m} \stackrel{\text { def }}{=} \prod_{\substack{n=1 \\ n \equiv r_{1}, \ldots, r_{t}(\bmod m)}}^{\infty}\left(1-x^{n}\right)
$$

and then clear the fractions, which gives the identity

$$
( \pm 1)_{5}( \pm 22)_{55}-x^{2}( \pm 2)_{5}( \pm 11)_{55}=( \pm 1, \pm 2)_{5}( \pm 11, \pm 22)_{55} \text {. }
$$

Multiplying through by $(0)_{5}(0)_{55}$ and rewriting, we obtain the identity

$$
\begin{gathered}
(0, \pm 22)_{55}(0, \pm 1)_{5}-x^{2}(0, \pm 11)_{55}(0, \pm 2)_{5} \\
=(0)_{1}(0)_{11}=(0, \pm 11)_{33}(0, \pm 1)_{3} .
\end{gathered}
$$

Identifying each factor in (3.2) as a certain $T_{1}(k, l)$, we obtain the desired $T^{2}$ identity, viz.

$$
T_{1}\left(\frac{55}{2}, \frac{11}{2}\right) T_{1}\left(\frac{5}{2}, \frac{3}{2}\right)-x^{2} T_{1}\left(\frac{55}{2}, \frac{33}{2}\right) T_{1}\left(\frac{5}{2}, \frac{1}{2}\right)=T_{1}\left(\frac{33}{2}, \frac{11}{2}\right) T_{1}\left(\frac{3}{2}, \frac{1}{2}\right) .
$$

(ii) We next use the expansion formula (cf. [4, p. 779]) (here we use the original notation) on each of the three terms in (3.3) to convert it into a balanced $T^{2}$ identity. Program Forward [4, p. 789] indicates that both $\left(k_{1}, k_{2}\right)=$ $\left(\frac{55}{2}, \frac{5}{2}\right)$ and $\left(k_{1}, k_{2}\right)=\left(\frac{33}{2}, \frac{3}{2}\right)$ can be expanded to the same pair $\left(K_{1}, K_{2}\right)=$ $(30,330)$. We therefore expand the two terms on the left side of (3.3) into sums that are balanced at $(30,330)$, using the parameters $[a, b, m]=[1,1,12]$ (cf. [4, p. 780$]$ ). This gives respectively the two groups of twelve terms in Table 1. Next, the term on the right of (3.3) is expanded using the parameters $[3,3,20]$, giving the twenty terms listed in Table 2 . (Note that the form of each term in all these expansions is $x^{\alpha_{i}} T_{0}\left(30, l_{1 i}\right) T_{0}\left(330, l_{2 i}\right)$, while in Tables 1 and 2 we have indicated only the signs and the values of $\alpha_{i}, l_{1 i}, l_{2 i}$.)

TABLE 1. Expansions using $[1,1,12]$

Group 1 terms

$T_{1}\left(\frac{55}{2}, \frac{11}{2}\right) T_{1}\left(\frac{5}{2}, \frac{3}{2}\right),[1,1,12]$

\begin{tabular}{|c|crrc|}
\hline$i$ & sign & $\alpha_{i}$ & $l_{1 i}$ & $l_{2 i}$ \\
\hline & & & & \\
1 & + & 0 & 4 & $22^{*}$ \\
2 & - & 1 & 9 & 33 \\
3 & - & 4 & 1 & 77 \\
4 & + & 7 & 14 & $88^{*}$ \\
5 & + & 13 & 6 & $132^{*}$ \\
6 & - & 18 & 19 & 143 \\
7 & - & 27 & 11 & 187 \\
8 & + & 34 & 24 & $198^{*}$ \\
9 & + & 46 & 16 & $242^{*}$ \\
10 & - & 55 & 29 & 253 \\
11 & - & 70 & 21 & 297 \\
12 & + & 77 & 26 & $308^{*}$ \\
\hline
\end{tabular}

Group 2 terms

$$
x^{2} T_{1}\left(\frac{55}{2}, \frac{33}{2}\right) T_{1}\left(\frac{5}{2}, \frac{1}{2}\right),[1,1,12]
$$

\begin{tabular}{|c|crrc|}
\hline$i$ & sign & $\alpha_{i}$ & $l_{1 i}$ & $l_{2 i}$ \\
\hline & & & & \\
1 & + & 2 & 16 & 22 \\
2 & - & 4 & 21 & 33 \\
3 & - & 5 & 11 & 77 \\
4 & + & 11 & 26 & 88 \\
5 & + & 13 & 6 & $132 *$ \\
6 & - & 22 & 29 & 143 \\
7 & - & 26 & 1 & 187 \\
8 & + & 34 & 24 & $198 *$ \\
9 & + & 44 & 4 & 242 \\
10 & - & 51 & 19 & 253 \\
11 & - & 67 & 9 & 297 \\
12 & + & 73 & 14 & 308 \\
\hline
\end{tabular}


TABLE 2. Expansion of $T_{1}\left(\frac{33}{2}, \frac{11}{2}\right) T_{1}\left(\frac{3}{2}, \frac{1}{2}\right)$ using $[3,3,20]$

Group 3 positive terms

\begin{tabular}{|c|rrc|}
\hline$i$ & $\alpha_{i}$ & $l_{1 i}$ & $l_{2 i}$ \\
\hline & & & \\
1 & 0 & 4 & $22 *$ \\
2 & 5 & 22 & 44 \\
3 & 7 & 14 & $88^{*}$ \\
4 & 12 & 20 & 110 \\
5 & 23 & 2 & 176 \\
6 & 24 & 28 & 154 \\
7 & 37 & 10 & 220 \\
8 & 46 & 16 & $242^{*}$ \\
9 & 62 & 8 & 286 \\
10 & 77 & 26 & $308^{*}$ \\
& & & \\
\hline
\end{tabular}

Group 3 negative terms

\begin{tabular}{|c|rrr|}
\hline$i$ & $\alpha_{i}$ & $l_{1 i}$ & $l_{2 i}$ \\
\hline 1 & 1 & 13 & 11 \\
2 & 2 & 5 & 55 \\
3 & 11 & 29 & 77 \\
4 & 15 & 23 & 121 \\
5 & 16 & 11 & 143 \\
6 & 29 & 19 & 187 \\
7 & 33 & 7 & 209 \\
8 & 48 & 1 & 253 \\
9 & 62 & 25 & 275 \\
10 & 79 & 17 & 319 \\
& & & \\
\hline
\end{tabular}

TABLE 3. $A_{i}(x)$ and $B_{i}(x)$

$A_{i}(x)=x^{\alpha_{i}} T\left(30, l_{1 i}\right) T\left(330, l_{2 i}\right)$

\begin{tabular}{|c|rrr|}
\hline$i$ & $\alpha_{i}$ & $l_{1 i}$ & $l_{2 i}$ \\
\hline 1 & 0 & 13 & 11 \\
2 & 1 & 5 & 55 \\
3 & 3 & 21 & 33 \\
4 & 4 & 11 & 77 \\
5 & 10 & 29 & 77 \\
6 & 14 & 23 & 121 \\
7 & 15 & 11 & 143 \\
8 & 21 & 29 & 143 \\
9 & 25 & 1 & 187 \\
10 & 28 & 19 & 187 \\
11 & 32 & 7 & 209 \\
12 & 47 & 1 & 253 \\
13 & 50 & 19 & 253 \\
14 & 61 & 25 & 275 \\
15 & 66 & 9 & 297 \\
16 & 78 & 17 & 319 \\
& & & \\
\hline
\end{tabular}

$B_{i}(x)=x^{\alpha_{i}} T\left(30, l_{1 i}\right) T\left(330, l_{2 i}\right)$

\begin{tabular}{|c|rrr|}
\hline$i$ & $\alpha_{i}$ & $l_{1 i}$ & $l_{2 i}$ \\
\hline & & & \\
1 & 0 & 9 & 33 \\
2 & 1 & 16 & 22 \\
3 & 3 & 1 & 77 \\
4 & 4 & 22 & 44 \\
5 & 10 & 26 & 88 \\
6 & 11 & 20 & 110 \\
7 & 17 & 19 & 143 \\
8 & 22 & 2 & 176 \\
9 & 23 & 28 & 154 \\
10 & 26 & 11 & 187 \\
11 & 36 & 10 & 220 \\
12 & 43 & 4 & 242 \\
13 & 54 & 29 & 253 \\
14 & 61 & 8 & 286 \\
15 & 69 & 21 & 297 \\
16 & 72 & 14 & 308 \\
& & & \\
\hline
\end{tabular}

Equation (3.3) now becomes

Group 1 terms - Group 2 terms = Group 3 terms . 
Four terms in Group 1 cancel across this equation with four positive terms in Group 3 and two terms in Group 1 cancel the two negative terms in Group 2. (These are marked with an asterisk in Tables 1 and 2.) This leaves 16 terms on each side of (3.4). Transposing the negative terms that remain in (3.4) to the other side of the equation and dividing out the factor $x$ in all the terms, we obtain the desired balanced $T^{2}$ identity:

$$
\sum_{i=1}^{16} A_{i}(x)=\sum_{i=1}^{16} B_{i}(x)
$$

where the $A_{i}(x)$ and $B_{i}(x)$ are listed in Table 3 . It is this equation we must prove to establish (3.1).

(iii) We now obtain three identities, given in Table 4 below, by choosing three sets of values for the parameters $m, k, u, v, e$, and $f$ in (2.4). The first two identities have $m=6, k=30, u=v=1$, and $(e, f)=\left(\frac{7}{2},-\frac{11}{2}\right)$ and $\left(\frac{9}{2}, \frac{33}{2}\right)$, respectively. The third identity has $m=10, k=10, u=v=3$, and $(e, f)=\left(\frac{5}{2}, \frac{11}{2}\right)$.

TABLE 4. The three identities with $k_{1}=30$ and $k_{2}=330$

Identity 1: $m=6, k=30, u=v=1, e=\frac{7}{2}, f=-\frac{11}{2}$

\begin{tabular}{|r|rrr|}
\hline$\#$ & $\alpha$ & $l_{1}$ & $l_{2}$ \\
\hline$*$ & 0 & 2 & 44 \\
$*$ & 3 & 12 & 66 \\
$*$ & 17 & 8 & 154 \\
$*$ & 26 & 22 & 176 \\
$*$ & 54 & 18 & 264 \\
$*$ & 67 & 28 & 286 \\
\hline
\end{tabular}

\begin{tabular}{|c|rrr|}
\hline$\#$ & $\alpha$ & $l_{1}$ & $l_{2}$ \\
\hline & & & \\
1 & 0 & 9 & 33 \\
3 & 3 & 1 & 77 \\
7 & 17 & 19 & 143 \\
10 & 26 & 11 & 187 \\
13 & 54 & 29 & 253 \\
15 & 69 & 21 & 297 \\
& & & \\
\hline
\end{tabular}

Identity 2: $m=6, k=30, u=v=1, e=\frac{9}{2}, f=\frac{33}{2}$

\begin{tabular}{|c|rrr|}
\hline$\#$ & $\alpha$ & $l_{1}$ & $l_{2}$ \\
\hline 3 & 0 & 21 & 33 \\
4 & 1 & 11 & 77 \\
8 & 18 & 29 & 143 \\
9 & 22 & 1 & 187 \\
13 & 47 & 19 & 253 \\
15 & 63 & 9 & 297 \\
& & & \\
\hline
\end{tabular}

\begin{tabular}{|c|rrr|}
\hline$\#$ & $\alpha$ & $l_{1}$ & $l_{2}$ \\
\hline$*$ & 0 & 12 & 66 \\
4 & 1 & 22 & 44 \\
8 & 19 & 2 & 176 \\
9 & 20 & 28 & 154 \\
$*$ & 51 & 18 & 264 \\
14 & 58 & 8 & 286 \\
& & & \\
\hline
\end{tabular}


Identity 3: $m=10, k=10, u=v=3, e=\frac{5}{2}, f=\frac{11}{2}$

\begin{tabular}{|c|rrr|}
\hline$\#$ & $\alpha$ & $l_{1}$ & $l_{2}$ \\
\hline & & & \\
1 & 0 & 13 & 11 \\
2 & 1 & 5 & 55 \\
5 & 10 & 29 & 77 \\
6 & 14 & 23 & 121 \\
7 & 15 & 11 & 143 \\
10 & 28 & 19 & 187 \\
11 & 32 & 7 & 209 \\
12 & 47 & 1 & 253 \\
14 & 61 & 25 & 275 \\
16 & 78 & 17 & 319 \\
& & & \\
\hline
\end{tabular}

\begin{tabular}{|c|rrr|}
\hline$\#$ & $\alpha$ & $l_{1}$ & $l_{2}$ \\
\hline$*$ & 0 & 2 & 44 \\
2 & 1 & 16 & 22 \\
5 & 10 & 26 & 88 \\
6 & 11 & 20 & 110 \\
$*$ & 17 & 8 & 154 \\
$*$ & 26 & 22 & 176 \\
11 & 36 & 10 & 220 \\
12 & 43 & 4 & 242 \\
$*$ & 67 & 28 & 286 \\
16 & 72 & 14 & 308 \\
& & & \\
\hline
\end{tabular}

Now form the following linear combination of the above three identities,

$$
\text { Identity } 1+x^{3} \times \text { Identity } 2+\text { Identity } 3 \text {, }
$$

which yields equation (3.5) when six terms on the left-hand side of Identity 1 are cancelled with six terms on the right-hand side of Identities 2 and 3. (The terms that cancel are indicated in the tables by an asterisk. The term numbers in the \# columns for the left and right sides in Table 4 correspond respectively to those in the " $i$ " columns in Table 3. Note that the $\alpha$-values in Identity 2 must be increased by 3 because of the multiplier $x^{3}$.)

\section{Two Balanced $Q^{2}$ IDENTities}

Within the set of balanced $T^{2}$ identities there is a special and interesting subset-the balanced $Q^{2}$ identities. (Here $Q$ stands for the usual quintuple product

$$
Q(m, k) \stackrel{\text { def }}{=} \prod_{n \in S}\left(1-x^{n}\right)=\sum_{-\infty}^{\infty} x^{m\left(3 n^{2}+n\right) / 2}\left(x^{-3 k n}-x^{3 k n+k}\right),
$$

where $\left.S=\left\{n \in Z^{+}: n \equiv 0, \pm k, \pm(m-2 k), \pm(m-k), m(\bmod 2 m)\right\}.\right)$

The two identities of this kind which we will prove in this section are trinomials of similar form. They have similar proofs as well, but are of quite different types, as we will show in another paper. Because these identities are already balanced, there is no need to balance them as we did in the proof of Theorem 2. At present, these are two of the three balanced $Q^{2}$ identities we know.

(a) The first of these identities was originally proved as part of a proof in [4, equation (16)]. To our knowledge, this is the first example of a balanced $Q^{2}$ identity to appear in the literature.

Theorem 3. We have that

$$
Q(8,3) Q(56,7)+x^{3} Q(8,1) Q(56,21)=Q(8,2) Q(56,14) \text {. }
$$


Proof. Since (4.1) is a $Q^{2}$ identity (balanced at $(8,56)$ ), we can routinely rewrite it as a $T^{2}$ identity (balanced at $(12,84)$ ) by first transforming each $Q$ in (4.1) into $T$ terms by the following formula [4, equation (24)]:

$$
Q(m, k)=T\left(\frac{3 m}{2}, \frac{m}{2}-3 k\right)-x^{k} T\left(\frac{3 m}{2}, \frac{m}{2}+3 k\right) .
$$

Therefore, using (2.1) and (2.2), we have

$$
Q(8,3)=T(12,5)-x^{2} T(12,11), \quad Q(8,1)=T(12,1)-x T(12,7),
$$

and

$$
Q(8,2)=T(12,2)-x^{2} T(12,10) .
$$

Replacing $x$ by $x^{7}$ in these three results, we obtain

$$
\begin{aligned}
Q(56,21) & =T(84,35)-x^{14} T(84,77), \\
Q(56,7) & =T(84,7)-x^{7} T(84,49),
\end{aligned}
$$

and

$$
Q(56,14)=T(84,14)-x^{14} T(84,70) .
$$

Thus, the first term in (4.1) becomes

$$
\begin{aligned}
Q(8,3) Q(56,7)= & {\left[T(12,5)-x^{2} T(12,11)\right]\left[T(84,7)-x^{7} T(84,49)\right] } \\
= & S(0,5,7)-S(2,11,7)-S(7,5,49) \\
& +S(9,11,49)
\end{aligned}
$$

where for simplicity we have written

$$
S\left(\alpha, l_{1}, l_{2}\right)=x^{\alpha} T\left(12, l_{1}\right) T\left(84, l_{2}\right) .
$$

If we expand the second and third terms in (4.1) in the same way, we obtain $x^{3} Q(8,1) Q(56,21)=S(3,1,35)-S(4,7,35)-S(17,1,77)+S(18,7,77)$ and

$Q(8,2) Q(56,14)=S(0,2,14)-S(2,10,14)-S(14,2,70)+S(16,10,70)$.

With the help of these three results, identity (4.1) becomes the balanced $T^{2}$ identity

$$
\begin{aligned}
& S(0,5,7)+S(2,10,14)+S(3,1,35)+S(9,11,49) \\
& \quad+S(14,2,70)+S(18,7,77) \\
&= S(0,2,14)+S(2,11,7)+S(4,7,35)+S(7,5,49) \\
&+S(16,10,70)+S(17,1,77) .
\end{aligned}
$$

To prove (4.4), we use the subsidiary identity derived from Theorem 1 by setting $m=4, k=12$, and $u=v=1$, viz.

$$
\begin{aligned}
\sum_{n=0}^{3} S & \left(6 n^{2}+2 e n, 6 n+e+f, 7(6 n+e)-f\right) \\
& =\sum_{n=0}^{3} S\left(6 n^{2}+2 e n, 6 n+e-f, 7(6 n+e)+f\right) .
\end{aligned}
$$


If we put $(e, f)=\left(\frac{3}{2}, \frac{7}{2}\right)$ and $(e, f)=\left(-\frac{1}{2}, \frac{21}{2}\right)$ into (4.5), we obtain respectively the two identities

$$
\begin{aligned}
& S(0,5,7)+S(3,1,35)+S(9,11,49)+S(18,7,77) \\
& \quad=S(0,2,14)+S(3,8,28)+S(9,4,56)+S(16,10,70)
\end{aligned}
$$

and

$$
\begin{aligned}
& S(0,10,14)+S(1,8,28)+S(7,4,56)+S(12,2,70) \\
& \quad=S(0,11,7)+S(2,7,35)+S(5,5,49)+S(15,1,77) .
\end{aligned}
$$

Then the linear combination $(4.6)+x^{2} \times(4.7)$ of these equations produces (4.4) when the common sum $S(3,8,28)+S(9,4,56)$ is cancelled from its two sides. (Note that multiplying an $S$ by $x^{n}$ increases the first argument of $S$ by $n$.)

Remark. The proof of (4.1) provides a third proof of identity (9) in [4].

(b) In the summer of 1990 we sent identity (4.1) to Oliver Atkin. Within 24 hours he sent us identity (4.8) below, another trinomial identity with a form similar to that of (4.1). An impressive performance!

Theorem 4 [Atkin]. We have that

$$
Q(14,3) Q(70,5)+x^{3} Q(14,1) Q(70,25)=Q(14,5) Q(70,15) .
$$

Proof. As in the proof of (4.1), we first use (4.2) to transform (4.8) into a $T^{2}$ equation, balanced at $(21,105)$. We find that

$$
Q(14,3)=T(21,2)-x^{3} T(21,16), \quad Q(14,1)=T(21,4)-x T(21,10),
$$

and

$$
Q(14,5)=T(21,8)-x^{4} T(21,20),
$$

and, replacing $x$ by $x^{5}$ in these results, we obtain the equations

$$
\begin{aligned}
Q(70,15) & =T(105,10)-x^{15} T(105,80), \\
Q(70,5) & =T(105,20)-x^{5} T(105,50),
\end{aligned}
$$

and

$$
Q(70,25)=T(105,40)-x^{20} T(105,100) .
$$

Thus, the three terms in (4.8) transform into

$$
\begin{aligned}
Q(14,3) Q(70,5)=S(0,2,20)-S(3,16,20) & \\
- & S(5,2,50)+S(8,16,50) \\
x^{3} Q(14,1) Q(70,25)= & S(3,4,40)-S(4,10,40) \\
& -S(23,4,100)+S(24,10,100),
\end{aligned}
$$

and

$$
\begin{aligned}
Q(14,5) Q(70,15)= & S(0,8,10)-S(4,20,10) \\
& -S(15,8,80)+S(19,20,80),
\end{aligned}
$$

where we have written

$$
S\left(\alpha, l_{1}, l_{2}\right)=x^{\alpha} T\left(21, l_{1}\right) T\left(105, l_{2}\right) .
$$


Thus, equation (4.8) becomes the balanced $T^{2}$ equation

$$
\begin{aligned}
& S(0,2,20)+S(3,4,40)+S(4,20,10)+S(8,16,50) \\
& \quad+S(15,8,80)+S(24,10,100) \\
&= S(0,8,10)+S(3,16,20)+S(4,10,40)+S(5,2,50) \\
&+S(19,20,80)+S(23,4,100) .
\end{aligned}
$$

Again we derive a subsidiary identity from Theorem 1 by setting $m=3$, $k=21$, and $u=v=1$, viz.

$$
\begin{aligned}
\sum_{n=0}^{2} S & \left(14 n^{2}+2 e n, 14 n+e+f, 5(14 n+e)-f\right) \\
= & \sum_{n=0}^{2} S\left(14 n^{2}+2 e n, 14 n+e-f, 5(14 n+e)+f\right) .
\end{aligned}
$$

Putting $(e, f)=(3,-5),(6,-10),(5,15)$ into (4.13) gives respectively the family of three identities

$$
\begin{aligned}
& S(0,2,20)+S(8,16,50)+S(20,12,90) \\
& \quad=S(0,8,10)+S(8,6,60)+S(19,20,80), \\
& S(0,4,40)+S(2,18,30)+S(21,10,100) \\
& \quad=S(0,16,20)+S(2,2,50)+S(17,12,90),
\end{aligned}
$$

and

$$
\begin{aligned}
& S(0,20,10)+S(4,6,60)+S(11,8,80) \\
& \quad=S(0,10,40)+S(1,18,30)+S(19,4,100) .
\end{aligned}
$$

The linear combination $(4.14)+x^{3} \times(4.15)+x^{4} \times(4.16)$ of these equations gives an identity which becomes $(4.12)$ when the common sum $S(5,18,30)+$ $S(8,6,60)+S(20,12,90)$ is cancelled from its two sides.

\section{ACKNOWLEDGMENT}

We would like to thank Greg Manning for his help in developing the algorithms that were used to find the proofs in this paper.

\section{BIBLIOGRAPHY}

1. B. J. Birch, A look back at Ramanujan's notebooks, Math. Proc. Cambridge Philos. Soc. 78 (1975), 73-79.

2. R. Blecksmith, J. Brillhart, and I. Gerst, Parity results for certain partition functions and identities similar to theta function identities, Math. Comp. 48 (1987), 29-38.

3. __ Some infinite product identities, Math. Comp. 51 (1988), 301-314.

4. $\ldots$ On a certain $(\bmod 2)$ identity and a method of proof by expansion, Math. Comp. 56 (1991), 775-794.

5. Soc. 13 (1992), 504. 
6. D. Bressoud, Proof and generalization of certain identities conjectured by Ramanujan, $\mathrm{PhD}$ Thesis, Temple University, Philadelphia, PA, 1977.

7. S. Ramanujan, Algebraic relations between certain infinite products, Collected Papers of Srinivasa Ramanujan, Chelsea, New York, 1962, p. 231.

8. S. Robins, Arithmetic properties of modular forms, $\mathrm{PhD}$ Thesis, University of California, Los Angeles, CA, 1991.

9. L. J. Rogers, On a type of modular relation, Proc. London Math. Soc. (2) 19 (1921), 387-397.

10. G. N. Watson, Proof of certain identities in combinatory analysis, J. Indian Math. Soc. 20 (1933), 57-69.

Department of Mathematical Sciences, Northern Illinois University, DeKalb, IlliNOIS 60115

E-mail address: richard@math.niu.edu

Department of Mathematics, University of Arizona, Tucson, Arizona 85721

E-mail address: jdb@math.arizona.edu

Department of Applied Mathematics and Statistics, SUNy at Stony Brook, Stony BROOK, NEW YORK 11794 